\title{
Spinal Cord Editor's Page March 2007
}

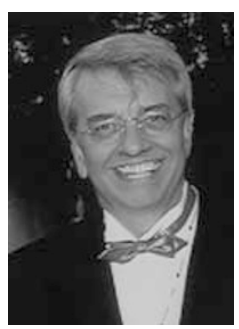

Dear Spinal Cord Reader,

The March issue of Spinal Cord contains four reports of the International Campaign for Cures of Spinal Cord Injury Paralysis (ICCP). The ICCP supported an international panel tasked with reviewing the methodology for spinal cord injury (SCI) clinical trials and making recommendations on the conduct of future trials.

You will appreciate the importance of this work.

Experimental interventions developed to improve functional outcomes after spinal cord injury (SCI) are expanding, and several of these will undergo clinical trials in the near future. In this series of articles, the International SCI Clinical Trials Guidelines Panel investigate four key areas and focus their initial recommendations on the design of clinical trials for experimental cell-based and pharmaceutical drug treatments for protection or repair of the injured spinal cord. They look at both acute and chronic stages of SCI. This focus is necessary because of the substantial risks and potential benefits of these types of treatment, and because some of these treatments have been offered without completing a clinical trial.

Ethical issues and requirements for evidence-based knowledge are two characteristics greatly valued in actual science and medical practice. Topics such as spontaneous recovery after SCI, statistical power needed for therapeutic clinical trials, outcome measures, inclusion/exclusion criteria, ethics, and trial design are all to be found in these reports.

These guidelines will be extremely valuable for the conduction of future clinical research. Their publication in Spinal Cord discloses them to a large specialized readership worldwide, and we expect thee guidelines to be quickly accepted as a standard approach.

In addition to the guidelines, you will find an original article on health behaviour in patients with SCI. As secondary impairments are prevalent among people with SCI and education on a healthy lifestyle is a core component of every rehabilitation programme, it is of great importance to rigorously test the efficacy of health behaviours promoted during rehabilitation. Longitudinal research is recommended.

Finally, two special Case Reports conclude this month's issue of Spinal Cord.

Spinal Cord (2007) 45, 189. doi:10.1038/sj.sc.3102046 